\title{
Caracterización de Estilos de Liderazgo mediante el Análisis de Redes Sociales
}

\section{Resumen}

El estudio presenta un análisis de redes para caracterizar los estilos de liderazgo en una institución de voluntariado, complementario o alternativo a los cuestionarios clásicos para medir el liderazgo. El estudio plantea preguntas reactivas para identificar las relaciones de amistad y los líderes destacados en las dimensiones del liderazgo transformacional, transaccional y pasivo/evitador y analiza, para cada una de ellas, las métricas de la estructura de la red en su conjunto y el rol individual de cada actor. El estudio a nivel exploratorio, basado en la opinión de 9 integrantes de un proyecto específico, permitió mostrar las bondades del análisis de redes aplicado al tema de liderazgo: (i) se identificó que el clima de "respeto y confianza", el "entusiasmo" y la "preocupación por el bienestar de las personas" predominan en la organización; y (ii) se identificó el rol individual de cada líder, se identificaron tres líderes que son los más amigos y se preocupan por el bienestar de los demás, pero uno de ellos destaca por transmitir "mayor respeto y confianza" y es "un ejemplo a seguir"; mientras que los otros dos destacan por ser los líderes más "entusiastas y optimistas" y "promueven la innovación y creatividad", entre otros hallazgos.

\section{Palabras clave:}

Liderazgo, Análisis de Redes, Gestión del Capital Humano. 


\section{Introducción}

El liderazgo institucional es uno de los temas más analizado en la Administración de Empresas, debido a los cambios organizacionales en respuesta a los cambios del entorno. Las relaciones dentro de las organizaciones que antes se basaban en la autoridad, la obediencia y las obligaciones contractuales ${ }^{1}$, han ido cambiando por la delegación, la colaboración y la participación²(Kausel \& Manzi, 2010).

Históricamente, el liderazgo ha sido ampliamente estudiado desde varias perspectivas y se han identificado varias dimensiones o aspectos relevantes que explican el liderazgo en las organizaciones.

Por otro lado, en diversas disciplinas se está utilizando el análisis de redes sociales para tratar de explicar las relaciones o interacciones entre los actores que conforman una red. En particular, y aplicado al tema de liderazgo, el análisis de redes sociales puede explicar cómo se forman las redes sociales alrededor de un líder y cómo éste afecta la conducta de las personas.

El presente artículo analiza a nivel exploratorio las redes sociales que conforman las diferentes dimensiones de liderazgo. Es por ello que se plantea el objetivo de caracterizar los estilos de liderazgo predominantes en una organización de voluntariado mediante el análisis de las redes sociales.

\section{Marco Teórico}

El liderazgo puede ser definido como la capacidad de una persona de influir en otras personas $\mathrm{y}$, desde esta perspectiva, el líder debe poseer ciertos rasgos 0 características que permiten influir en las personas. Desde otra perspectiva, el liderazgo puede ser visto como el proceso mediante el cual una persona líder ejerce poder sobre otras personas. Bajo este enfoque, para explicar el liderazgo también es necesario incluir y analizar el contexto y las percepciones y atribuciones de los seguidores(Sternberg, Antonakis, \& Ciancio, 2004).

Robert House y James MacGregor Burns plantearon la idea de un liderazgo carismático y transformacional. Sin embargo, Bernard M. Bass se basó en ideas previas para poder formular el enfoque transformacional tomando en cuenta tanto rasgos y conductas del líder como variables situacionales (Lupano Perugini \& Castro Solari, 2008).

\footnotetext{
${ }^{1}$ Los autores citan alvancevich, J; Konopaske, R. (2010), “Organizational Behavior and Management”, (9a ed.), McGraw-Hill/Irwin

2 Los autores citan a Walton, R.E. (1985), "From control to comitment into the workplace”, Harvard Business Review", Vol 63(2), pp. 77-84
} 
El liderazgo transformacional (Bass, 1985, 1990; Burns, 1978) ${ }^{3}$ es la capacidad de inspirar y motivar a los subordinados para que logren resultados, identificándose las siguientes dimensiones para explicar el liderazgo:

o Influencia Idealizada Atribuida: los líderes demuestran conductas éticas y morales, promueven que los seguidores se identifiquen con ellos (Bracho, García, \& Jiménez, 2012).

o Influencia Idealizada Conductual: los líderes muestran conductas que sirven como modelo para sus colegas (Munaf, 2011).

o Motivación e Inspiración: los líderes fomentan el espíritu de grupo y generan expectativas de futuro (Bracho, García, \& Jiménez, 2012).

o Estimulación intelectual: los líderes promueven esfuerzos para plantear ideas innovadoras y creativas (Bracho, García, \& Jiménez, 2012).

- Consideración Individual: los líderes prestan atención a las necesidades individuales de desarrollo de cada seguidor (Bracho, García, \& Jiménez, 2012).

0

El liderazgo transaccional se basa en el intercambio de desempeño por recompensas o castigos y tiene fundamentalmente las siguientes dimensiones:

- Recompensa Contingente: intercambian y la negocian con los subordinados con el fin de lograr objetivos (Kausel \& Manzi, 2010; Nader \& Castro Solano, 2007).

o Dirección por Excepción Activa: los líderes se centran en corregir los errores con el fin de fallos y cumplir los objetivos organizacionales (Almería, 2010).

Bass y Riggio (2006) afirman que el modelo de liderazgo ha evolucionado hacia el Liderazgo de Rango Completo (Full Range Leadership) que, además de las dimensiones del liderazgo transformacional y transaccional, incluye la dimensión Laissez-Faire, aspecto más negativo del liderazgo. En este enfoque denominado pasivo/evitador, algunos autores también proponen incluir la dimensión Dirección por Excepción Pasiva:

o Dirección por Excepción Pasiva: los líderes solo actúan cuando se presentan dificultades o problemas (Universidad Militar Nueva Granada, 2006).

o Laissez-Faire (dejar hacer): los líderes evitan tomar decisiones, no hacen uso de la autoridad que el rol de líder les confiere, (Nader \& Castro Solano, 2007).

\footnotetext{
3 Bass, B (1985), “Leadership and Performance Beyond Expectations”, New York: Free Press; Bass, B. (1990), "From transactional to transformational Leadership: Learning to share a vision", en R.Steers, y G. Bigley (Eds.), Motivation and Leadership at Work, pp. 346-354, New York: McGraw-Hill; Burns, J.M. (1978), "Leadership", New York, NY: Harper \& Row.
} 
Por otro lado, los científicos sociales utilizan los conceptos asociados al análisis de redes para el estudio de asuntos variados (Sanz Menéndez, 2003), se utiliza en sociología, antropología, ciencias políticas, gestión organizacional, medios de comunicación y otras disciplinas (Monsalve Moreno, 2008).

Una red social es un conjunto de actores o personas vinculados entre sí por algún motivo específico como amistad, poder, alianzas, parentesco o afecto, entre otras (Monsalve Moreno, 2008). El análisis de redes sociales, ARS (social network analysis), es una herramienta gráfica y de medición y análisis de las conductas de los individuos a nivel micro, y la estructura de la red a nivel macro (Sanz Menéndez, 2003), bajo el supuesto estos dos niveles permiten una mejor comprensión del fenómeno en estudio (Lugo Morin, 2011)(Sanz Menéndez, 2003). Las Métricas de Centralidad de cada actor comúnmente utilizadas en el análisis de redes sociales son: Grado ("Degree"), Cercanía ("Closeness"), Intermediación("Betweenness"), Vector Propio("Eingenvector") y el Índice de poder de Bonacich ("Bonacich's power"); mientras que las principales Métricas de la estructura de la red en su conjunto son: "Densidad", "Centralización" y "Unipolaridad".

Las redes sociales también pueden utilizarse para analizar la estructura de relaciones de liderazgo en un grupo social. Mendoza (2008) diseñó un cuestionario formulando una pregunta para medir las dimensiones de liderazgo establecidas por Bass y Avolio: "Amistad, grupo de pertenencia": relaciones de amistad existentes entre los integrantes del grupo; "Influencia idealizada atribuida": líderes que tienen el respeto y la confianza del grupo; "Influencia idealizada observada": líderes que son considerados por el grupo como un ejemplo a seguir; "Motivación e Inspiración": líderes que inyectan entusiasmo, optimismo y estándares de excelencia; "Estímulo Intelectual”: líderes que estimulan la innovación y la creatividad en el grupo; "Consideración Individual": líderes que se preocupan por el bienestar de los integrantes del grupo y les brindan apoyo; "Indiferencia": identificación de integrantes formales del grupo que no están integrados o están débilmente integrados a este; y "Amistad, grupo de referencia": líderes que impactan y movilizan hacia ellos a integrantes del grupo. En este estudio (Mendoza Torres, 2008) se incluyeron variables adicionales a las que suelen asociarse al liderazgo: amistad en grupos de pertenencia y de referencia y la "indiferencia".

\section{Metodología}

Considerando las dimensiones de liderazgo identificadas en la revisión de la literatura y el cuestionario de medición propuesto por Mendoza (2008) basado en el análisis de redes, para el presente estudio se planteó la siguiente estructura y cuestionario: 
Cuadro 1: Estructura del cuestionario

\begin{tabular}{|c|c|c|c|}
\hline \multicolumn{2}{|r|}{ Dimensión de Liderazgo } & \multicolumn{2}{|c|}{ Pregunta del Cuestionario: Líderes que ... } \\
\hline \multicolumn{2}{|l|}{ Amistad } & 1 & ... tienen mayor amistad conmigo \\
\hline \multirow{5}{*}{ 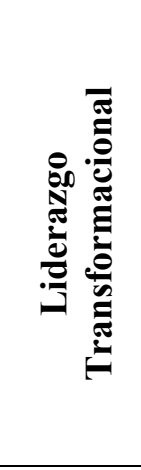 } & Influencia Idealizada Atribuida & 2 & ... tienen el respeto y la confianza del grupo \\
\hline & $\begin{array}{l}\text { Influencia Idealizada } \\
\text { Conductual }\end{array}$ & 3 & $\begin{array}{l}\text {... son considerados por el grupo como un } \\
\text { ejemplo a seguir }\end{array}$ \\
\hline & Motivación e Inspiración & 4 & $\begin{array}{l}\ldots \text { inyectan entusiasmo, optimismo y estándares } \\
\text { de excelencia al grupo }\end{array}$ \\
\hline & Estímulo Intelectual & 5 & $\begin{array}{l}\text {... estimulan la innovación y la creatividad en el } \\
\text { grupo }\end{array}$ \\
\hline & Consideración Individual & 6 & $\begin{array}{l}\text {... se preocupan por el bienestar de los } \\
\text { integrantes del grupo y les brindan apoyo }\end{array}$ \\
\hline \multirow{2}{*}{ 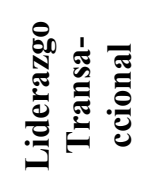 } & Recompensa Contingente & 7 & $\begin{array}{l}\text {... identifican las responsabilidades de los } \\
\text { demás y valoran o premian el logro de objetivos }\end{array}$ \\
\hline & Dirección por Excepción Activa & 8 & $\begin{array}{l}\text {...conversan los errores de los demás cuando no } \\
\text { se logran los resultados }\end{array}$ \\
\hline \multirow{2}{*}{ 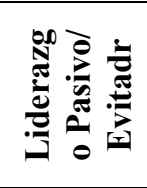 } & Dirección por Excepción Pasiva & 9 & $\begin{array}{l}\text {...sólo intervienen cuando los problemas se } \\
\text { vuelven serios }\end{array}$ \\
\hline & Laissez-faire & 10 & $\begin{array}{l}\text {... evitan tomar decisiones que puedan } \\
\text { implicarlos en asuntos importantes }\end{array}$ \\
\hline
\end{tabular}

Fuente: adaptado de (Mendoza Torres, 2008)

El diseño del cuestionario adoptó un formato matricial, en filas y columnas tal como se muestra a continuación, que permite que los actores encuestados marquen con aspa (“X”) a los que en su opinión se distinguen en cada pregunta:

\section{Cuadro 2: Diseño/Formato del cuestionario}

\begin{tabular}{|c|c|c|c|c|c|c|c|c|c|c|}
\hline \multirow[b]{2}{*}{ ACTOR } & \multicolumn{10}{|c|}{ Líderes que ... } \\
\hline & $\begin{array}{l}\text {... tienen mayor } \\
\text { amistad conmigo }\end{array}$ & $\begin{array}{l}\text { (Preg. } \\
2)\end{array}$ & $\begin{array}{l}\text { (Preg. } \\
3)\end{array}$ & $\begin{array}{l}\text { (Preg. } \\
4)\end{array}$ & $\begin{array}{l}\text { (Preg. } \\
5 \text { ) }\end{array}$ & $\begin{array}{l}\text { (Preg. } \\
6)\end{array}$ & $\begin{array}{l}\text { (Preg. } \\
7)\end{array}$ & $\begin{array}{l}\text { (Preg. } \\
8)\end{array}$ & $\begin{array}{l}\text { (Preg. } \\
9)\end{array}$ & $\begin{array}{c}\text { (Preg. } \\
10)\end{array}$ \\
\hline A1 & & $\mathbf{X}$ & $\mathbf{X}$ & & & $\mathbf{X}$ & & & $\mathbf{X}$ & \\
\hline & & & $\mathbf{X}$ & & & & $\mathbf{X}$ & & $\mathbf{X}$ & \\
\hline $\mathbf{A}_{\mathbf{i}}$ & & & & $\mathbf{X}$ & & & $\mathbf{X}$ & & & \\
\hline
\end{tabular}

Fuente: adaptado de (Mendoza Torres, 2008)

Para el estudio piloto, se encuestó a 9 miembros de una organización sin fines de lucro que realiza actividades sociales, del 29 de marzo al 05 de abril de 2014. La muestra compuesta mayormente por jóvenes voluntarios entre 22 y 34 años, fue elegida por el único criterio de explorar el liderazgo de los integrantes de un proyecto específico. 
Se empleó la técnica denominada "análisis de redes sociales", para la cual las respuestas del cuestionario se tabularon en matrices con variables binarias "uno" o "cero" según la existencia o no de la relación a medir:

Cuadro 4: Matriz de Relaciones de una Red - Ejemplo "AMISTAD"

\begin{tabular}{|c|c|c|c|}
\hline ACTOR & A1 & & $\mathbf{A}_{\mathbf{i}}$ \\
\hline $\mathbf{A 1}$ & & $\mathbf{0}$ & $\mathbf{0}$ \\
\hline & & & \\
\hline $\mathbf{A}_{\mathbf{i}}$ & $\mathbf{1}$ & $\mathbf{0}$ & \\
\hline
\end{tabular}

Fuente: elaboración propia en base a técnica "Análisis de Redes"

Se utilizó el software UCINET para graficar las relaciones entre los actores y el cálculo de las métricas correspondientes, para cada una de las 10 redes que representan cada una de las 10 dimensiones de liderazgo medidas a través de las preguntas del cuestionario. Referente a las métricas seleccionadas para el análisis, teniendo presente la naturaleza asimétrica de las matrices de datos, se consideraron las siguientes:

Cuadro 3: Métricas de Redes Sociales consideradas en el estudio

\begin{tabular}{|c|c|c|}
\hline $\begin{array}{l}\text { Nivel de } \\
\text { Medición }\end{array}$ & Métrica & Descripción \\
\hline \multirow{4}{*}{$\begin{array}{l}\text { Métricas de } \\
\text { Centralidad de } \\
\text { cada actor }\end{array}$} & Grado & Número de actores que reconocen la amistad o \\
\hline & (Degree) & $\begin{array}{l}\text { liderazgo de cada actor, en porcentaje respecto al } \\
\text { máximo posible (grado estandarizado). }\end{array}$ \\
\hline & $\begin{array}{l}\text { Índice de poder de } \\
\text { Bonacich }\end{array}$ & $\begin{array}{l}\text { Poder o influencia de cada actor sobre los actores que } \\
\text { reconocen su amistad o liderazgo. El actor con más } \\
\text { "poder" es aquel que tiene más influencia en el mayor }\end{array}$ \\
\hline & (Bonacich's power) & $\begin{array}{l}\text { número de miembros de la red y, a su vez, éstos } \\
\text { reconocen menos líderes. }\end{array}$ \\
\hline \multirow{2}{*}{$\begin{array}{l}\text { Métricas de la } \\
\text { estructura de } \\
\text { la red }\end{array}$} & Densidad & $\begin{array}{l}\text { Total de conexiones o relaciones de liderazgo en toda } \\
\text { la red, expresada en porcentaje respecto a todos los } \\
\text { posibles contactos. }\end{array}$ \\
\hline & Centralización & $\begin{array}{l}\text { Nivel de dependencia de la red con respecto al } \\
\text { liderazgo de un "actor central", el de mayor liderazgo } \\
\text { entre los actores. }\end{array}$ \\
\hline
\end{tabular}

Fuente: metodo del estudio

\section{Resultados}

A continuación se muestran los principales resultados de la estructura de red en su conjunto y el análisis individual de cada uno de los actores. Los gráficos 1 y 2 presentan las relaciones entre actores de cada una de las dimensiones definidas en el estudio, habiendo sido incluidas las sus métricas correspondientes en la tabla 1. 
Gráfico 1: Red de Amistad y dimensiones de Liderazgo Transformacional

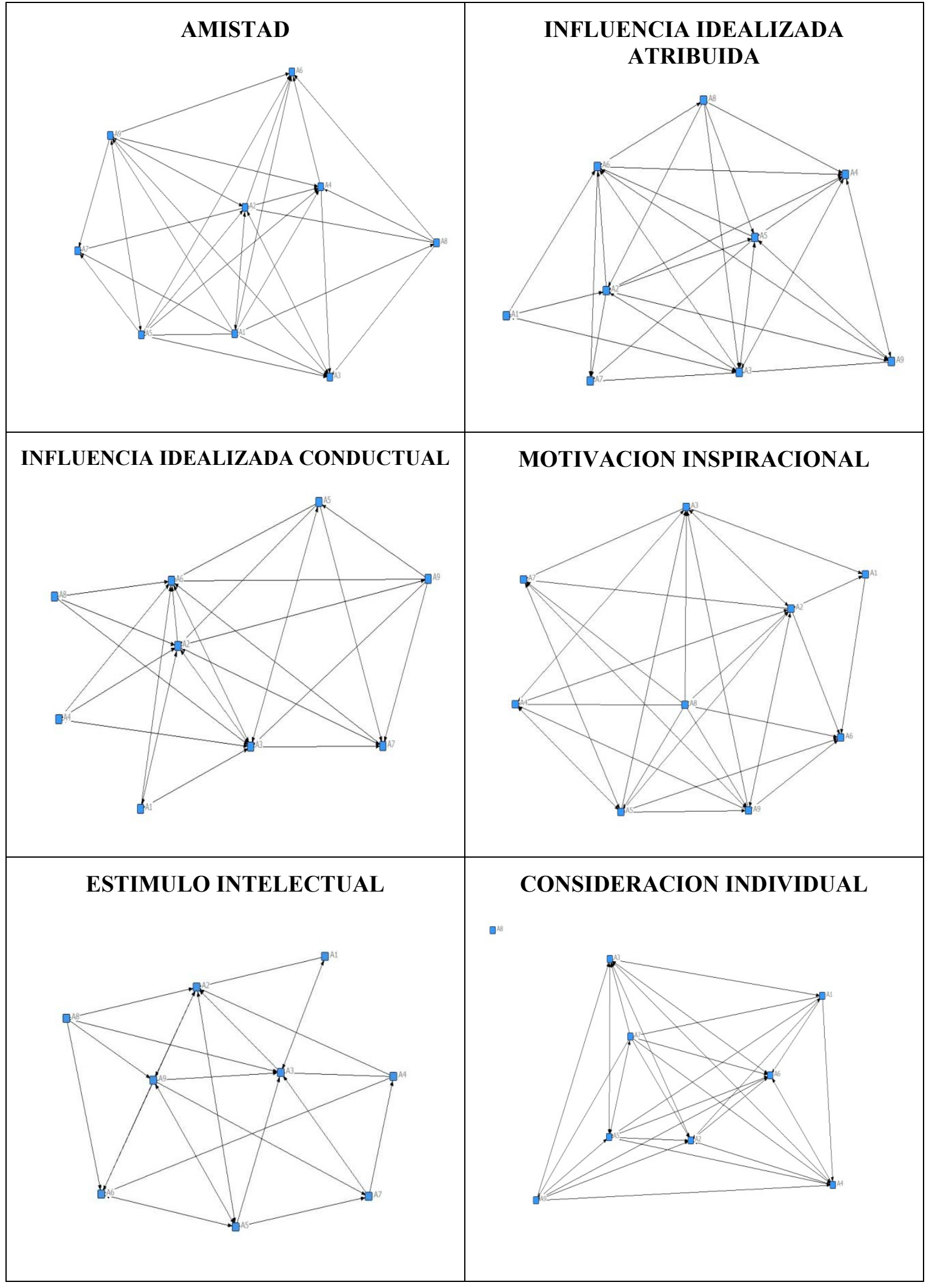

Fuente: datos del estudio procesados en el software UCINET 
Gráfico 2: Red de dimensiones de Liderazgo Transsaccional y Pasivo/Evitador

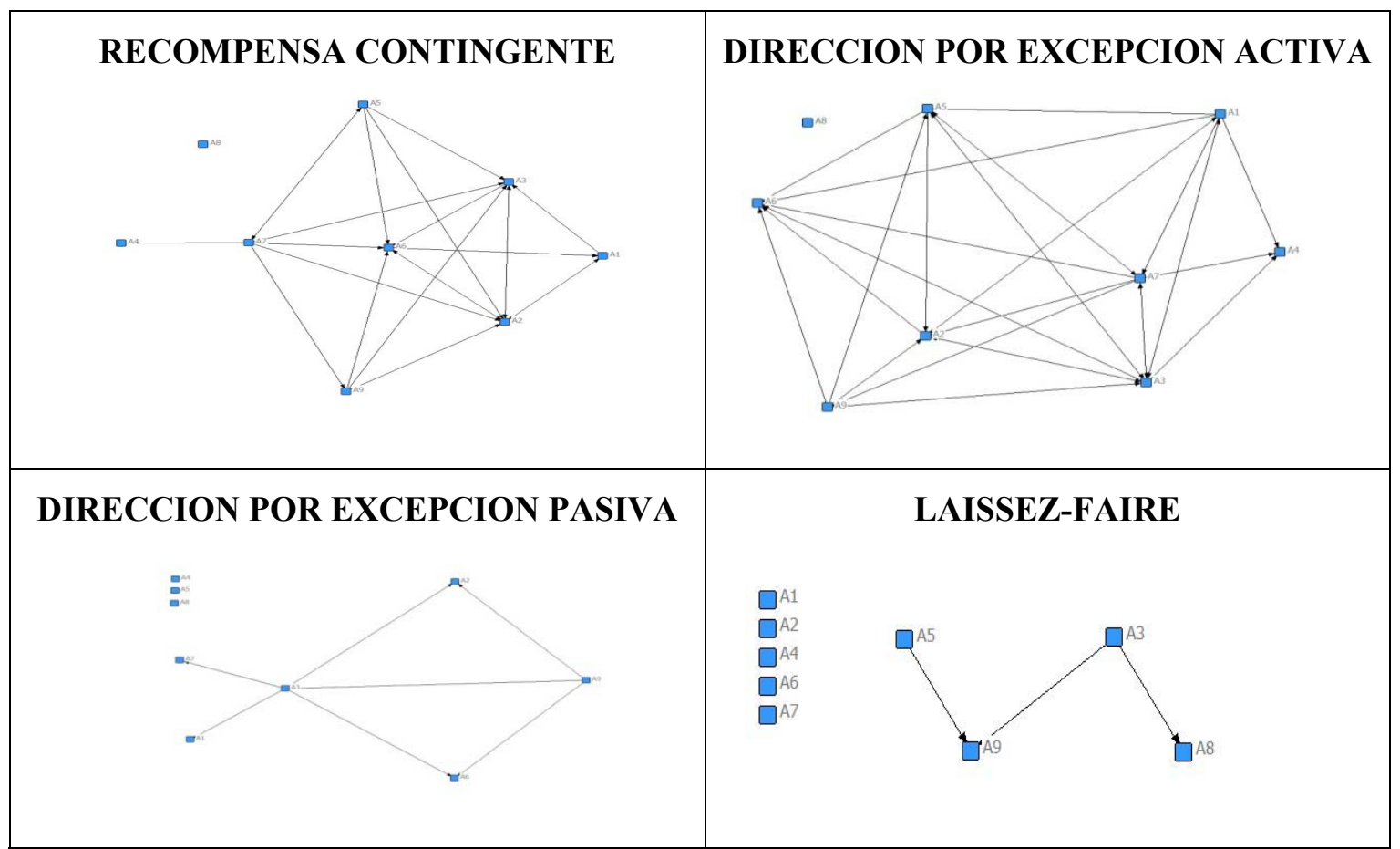

Fuente: datos del estudio procesados en el software UCINET

Tabla1: Métricas de la Estructura de la Red de Liderazgo

\begin{tabular}{|c|c|c|c|}
\hline \multicolumn{2}{|c|}{ Dimensión de Liderazgo } & $\begin{array}{c}\text { Densidad de la } \\
\text { Red }\end{array}$ & $\begin{array}{c}\text { Centralización } \\
\text { de la Red }\end{array}$ \\
\hline \multicolumn{2}{|l|}{ Amistad } & $48.6 \%$ & $29.7 \%$ \\
\hline \multirow{5}{*}{$\begin{array}{l}\text { Liderazgo } \\
\text { Transforma- } \\
\quad \text { cional }\end{array}$} & Influencia Idealizada Atribuida & $55.6 \%$ & $35.9 \%$ \\
\hline & Influencia Idealizada Conductual & $45.8 \%$ & $60.9 \%$ \\
\hline & Motivación e Inspiración & $51.4 \%$ & $26.6 \%$ \\
\hline & Estímulo Intelectual & $40.3 \%$ & $53.1 \%$ \\
\hline & Consideración Individual & $50.0 \%$ & $42.2 \%$ \\
\hline \multirow{2}{*}{$\begin{array}{c}\text { Liderazgo } \\
\text { Transaccional }\end{array}$} & Recompensa Contingente & $31.9 \%$ & $48.4 \%$ \\
\hline & Dirección por Excepción Activa & $40.3 \%$ & $39.1 \%$ \\
\hline $\begin{array}{l}\text { Liderazgo } \\
\text { Pasivo/ }\end{array}$ & Dirección por Excepción Pasiva & $9.7 \%$ & $17.2 \%$ \\
\hline Evitador & Laissez-Faire & $4.2 \%$ & $23.4 \%$ \\
\hline
\end{tabular}

Fuente: datos del estudio procesados en el software UCINET 
Los estilos predominantes de liderazgo en el grupo son "Influencia Idealizada Atribuida" (densidad=55.6\%), "Motivación e Inspiración" (densidad=51.4\%) y "Consideración Individual" (densidad=50.0\%). Es decir, los miembros de la red "tienen el respeto y la confianza del grupo", "inyectan entusiasmo y optimismo" y "se preocupan por el bienestar de los integrantes". En un segundo nivel, están las relaciones de "Amistad" (densidad=48.6\%) y que los miembros son "ejemplos a seguir" ("Influencia Idealizada Conductual", densidad=45.8\%). Un hallazgo importante del estudio es que los vínculos de amistad que fueron acuñados de manera adicional al a las variables que definen el liderazgo, tienen influencia en las dimensiones que definen el liderazgo transformacional.

Respecto a la centralización de las dimensiones, la "Influencia Idealizada Conductual" representa la de mayor grado (centralidad $=60.9 \%$ ); es decir, hay relativamente pocos actores que son vistos "como ejemplos a seguir". Por el contrario, el entusiasmo (dimensión "Motivación e Inspiración"; centralidad=26.56\%) y la amistad (centralidad $=29.69 \%$ ) son las características más compartidas en el grupo. La dimensión "Estímulo Intelectual" representa la de menor grado del liderazgo transformacional (densidad=40.3\%) y con una alta concentración en pocas personas (centralización $=53.13 \%$ ), muy pocos en la organización "estimulan la innovación y la creatividad en el grupo".

En niveles mucho más bajos de predominancia se encuentran las dimensiones que corresponden al "liderazgo transaccional". No son prácticas habituales que "los líderes conversan los errores de los demás cuando no se logran los resultados" de la dimensión "Dirección por Excepción Activa" (densidad=40.3\%; centralización=39.1\%); y que "los líderes valoran o premian el logro de objetivos", que corresponde a la dimensión "Recompensa Contingente" (densidad=31.9\%; centralización=48.4\%).

En un nivel mucho más bajo, las dimensiones que corresponden al "liderazgo pasivo/evitador" resultaron prácticas no habituales, aspecto muy positivo. "Los líderes sólo intervienen cuando los problemas se vuelven serios" que corresponde a la dimensión "Dirección por Excepción Pasiva" (densidad=9.7\%; centralización=23.4\%); y "los líderes evitan tomar decisiones que puedan implicarlos en asuntos importantes" que corresponde a la dimensión "Laissez-Faire" (densidad=4.2\%; centralización $=23.4 \%$ ) representan ausencia casi completa de estos aspectos de "no liderazgo".

\section{-Perfil de Liderazgo de Actores}

A continuación se muestran los resultados de las métricas de centralidad de actores. En la aabla 2 se puede apreciar el rol de cada uno de los actores en las redes de amistad y liderazgo. Los actor "A2", "A3" y "A6" son los considerados como más amigos (degree $=75.0 \%$, índice de poder=1.25), seguido del actor "A4" (degree=62.5\%; índice de poder=0.95). 
Tabla2: Métricas de Centralidad de cada Actor de la Red de Liderazgo

\begin{tabular}{|c|c|c|c|c|c|c|c|c|c|c|}
\hline \multirow{2}{*}{$\begin{array}{c}\text { ACT } \\
\text { OR }\end{array}$} & \multicolumn{2}{|c|}{ AMISTAD } & \multicolumn{2}{|c|}{ INF. ID. ATRIB. } & \multicolumn{2}{|c|}{ INF. ID. COND. } & \multicolumn{2}{|c|}{ MOTIVAC. INSP } & \multicolumn{2}{|c|}{ ESTIM.INTEL. } \\
\hline & $\begin{array}{c}\text { Grado } \\
\text { (Degree) }\end{array}$ & $\begin{array}{c}\text { Poder } \\
\text { Bonacich }\end{array}$ & $\begin{array}{c}\text { Grado } \\
\text { (Degree) }\end{array}$ & $\begin{array}{c}\text { Poder } \\
\text { Bonacich }\end{array}$ & $\begin{array}{c}\text { Grado } \\
\text { (Degree) }\end{array}$ & $\begin{array}{c}\text { Poder } \\
\text { Bonacich }\end{array}$ & $\begin{array}{c}\text { Grado } \\
\text { (Degree) }\end{array}$ & $\begin{array}{c}\text { Poder } \\
\text { Bonacich }\end{array}$ & $\begin{array}{c}\text { Grado } \\
\text { (Degree) }\end{array}$ & $\begin{array}{c}\text { Poder } \\
\text { Bonacich }\end{array}$ \\
\hline A1 & $12.5 \%$ & 0.38 & $25.0 \%$ & 0.52 & $25.0 \%$ & 0.71 & $25.0 \%$ & 0.67 & $12.5 \%$ & 0.27 \\
\hline A2 & $75.0 \%$ & 1.25 & $75.0 \%$ & 1.17 & $87.5 \%$ & 1.24 & $75.0 \%$ & 1.27 & $87.5 \%$ & 1.30 \\
\hline A3 & $75.0 \%$ & 1.25 & $75.0 \%$ & 1.17 & $87.5 \%$ & 1.38 & $75.0 \%$ & 1.27 & $75.0 \%$ & 0.90 \\
\hline A4 & $62.5 \%$ & 0.95 & $75.0 \%$ & 1.33 & $25.0 \%$ & 0.71 & $62.5 \%$ & 1.12 & $12.5 \%$ & 0.18 \\
\hline A5 & $37.5 \%$ & 0.63 & $62.5 \%$ & 1.07 & $25.0 \%$ & 0.47 & $50.0 \%$ & 0.83 & $37.5 \%$ & 0.99 \\
\hline A6 & $75.0 \%$ & 1.25 & $87.5 \%$ & 1.43 & $100 \%$ & 1.58 & $62.5 \%$ & 0.97 & $62.5 \%$ & 1.03 \\
\hline A7 & $50.0 \%$ & 0.92 & $50.0 \%$ & 1.07 & $50.0 \%$ & 0.93 & $62.5 \%$ & 1.12 & $25.0 \%$ & 0.59 \\
\hline A8 & $12.5 \%$ & 0.12 & $12.5 \%$ & 0.32 & $0.0 \%$ & 0.00 & $0.0 \%$ & 0.00 & $0.0 \%$ & 0.00 \\
\hline A9 & $37.5 \%$ & 0.70 & $37.5 \%$ & 0.87 & $12.5 \%$ & 0.34 & $50.0 \%$ & 0.86 & $50.0 \%$ & 0.96 \\
\hline
\end{tabular}

\begin{tabular}{|c|c|c|c|c|c|c|c|c|c|c|}
\hline \multirow{2}{*}{$\begin{array}{c}\text { ACT } \\
\text { OR }\end{array}$} & \multicolumn{2}{|c|}{ CONSID.INDIV. } & \multicolumn{2}{c|}{ RECOM.CONT. } & \multicolumn{2}{c|}{ DIR.EXC.ACT. } & \multicolumn{2}{c|}{ DIR.EXC.PAS. } & \multicolumn{2}{c|}{ LAISSEZ-FAIRE } \\
\hline $\begin{array}{c}\text { Grado } \\
\text { (Degree) }\end{array}$ & $\begin{array}{c}\text { Poder } \\
\text { Bonacich }\end{array}$ & $\begin{array}{c}\text { Grado } \\
\text { (Degree) }\end{array}$ & $\begin{array}{c}\text { Poder } \\
\text { Bonacich }\end{array}$ & $\begin{array}{c}\text { Grado } \\
\text { (Degree) }\end{array}$ & $\begin{array}{c}\text { Poder } \\
\text { Bonacich }\end{array}$ & $\begin{array}{c}\text { Grado } \\
\text { (Degree) }\end{array}$ & $\begin{array}{c}\text { Poder } \\
\text { Bonacich }\end{array}$ & $\begin{array}{c}\text { Grado } \\
\text { (Degree) }\end{array}$ & $\begin{array}{c}\text { Poder } \\
\text { Bonacich }\end{array}$ \\
\hline A1 & $25.0 \%$ & 0.77 & $25.0 \%$ & 1.19 & $25.0 \%$ & 0.61 & $\mathbf{1 2 . 5 \%}$ & 0.001 & $0.0 \%$ & 0.000 \\
\hline $\mathbf{A 2}$ & $\mathbf{8 7 . 5 \%}$ & $\mathbf{1 . 5 5}$ & $\mathbf{7 5 . 0} \%$ & $\mathbf{1 . 5 0}$ & $\mathbf{6 2 . 5 \%}$ & 1.00 & $\mathbf{2 5 . 0 \%}$ & 0.002 & $0.0 \%$ & 0.000 \\
\hline $\mathbf{A 3}$ & $\mathbf{8 7 . 5 \%}$ & $\mathbf{1 . 5 5}$ & $\mathbf{6 2 . 5 \%}$ & 1.07 & $\mathbf{6 2 . 5 \%}$ & 1.00 & $12.5 \%$ & 0.001 & $0.0 \%$ & 0.000 \\
\hline $\mathbf{A 4}$ & $\mathbf{6 2 . 5 \%}$ & 0.93 & $12.5 \%$ & 0.00 & $37.5 \%$ & 0.70 & $0.0 \%$ & 0.000 & $0.0 \%$ & 0.000 \\
\hline $\mathbf{A 5}$ & $50.0 \%$ & 0.74 & $12.5 \%$ & 0.00 & $50.0 \%$ & 0.77 & $0.0 \%$ & 0.000 & $0.0 \%$ & 0.000 \\
\hline $\mathbf{A 6}$ & $\mathbf{8 7 . 5 \%}$ & $\mathbf{1 . 5 5}$ & $\mathbf{7 5 . 0 \%}$ & $\mathbf{1 . 5 0}$ & $\mathbf{7 5 . 0 \%}$ & $\mathbf{1 . 3 0}$ & $\mathbf{2 5 . 0 \%}$ & 0.002 & $0.0 \%$ & 0.000 \\
\hline $\mathbf{A 7}$ & $25.0 \%$ & 0.38 & $12.5 \%$ & 0.00 & $37.5 \%$ & 0.72 & $12.5 \%$ & 0.001 & $0.0 \%$ & 0.000 \\
\hline $\mathbf{A 8}$ & $0.0 \%$ & 0.00 & $0.0 \%$ & 0.00 & $0.0 \%$ & 0.00 & $0.0 \%$ & 0.000 & $12.5 \%$ & 0.001 \\
\hline $\mathbf{A 9}$ & $25.0 \%$ & 0.28 & $12.5 \%$ & 0.00 & $12.5 \%$ & 0.22 & $0.0 \%$ & 0.000 & $25.0 \%$ & 0.002 \\
\hline
\end{tabular}

Fuente: datos del estudio procesados en el software UCINET

Sin embargo, el actor "A6" es que transmite mayor "respeto y confianza al grupo" ("Influencia Idealizada Atribuida"; degree=87.5\%; índice de poder=1.43) y está mejor considerado "como un ejemplo a seguir" ("Influencia Idealizada Conductual"; degree $=100 \%$, índice de poder=1.58). El segundo lugar de estas dimensiones lo ocupan los actores "A2" y "A3".

Por el contrario, los actores "A2" y "A3" son los que más "inyectan entusiasmo y optimismo" ("Motivación Inspiracional"; degree $=75 \%$ e índice de poder $=1.27$, ambos) y "estimulan la innovación y la creatividad en el grupo" ("Estimulación Intelectual"; degree $=87.5 \%$ y $75 \%$, índice de poder $=1.30$ y 0.90 , respectivamente). Aunque el actor "A6" está en tercer lugar en estas dimensiones, tiene "mayor poder" para promover la innovación y creatividad que el actor "A3".

En la dimensión "Consideración Individual", los tres actores más líderes, "A2", "A3" y "A6" son los que más "se preocupan por el bienestar de los integrantes del grupo y les brindan apoyo" (degree=87.5\%, índice de poder=1.55, en los tres actores), notándose que esta dimensión tiene un patrón de comportamiento similar al grado de amistad.

En todas las dimensiones descritas, que corresponden al liderazgo transformacional, han destacado los líderes "A6", "A2" y "A3". Los tres son los más amigos y se preocupan por el bienestar de los demás. El actor "A6" destaca por las dimensiones de 
Influencia Idealizada es el de mayor respeto, confianza y ejemplo a seguir. Los actores "A2" y "A3" son más entusiastas, optimistas y promueven la innovación y creatividad. Después de estos tres líderes, el actor "A4" asoma como líder en algunas dimensiones: amistad, entusiasmo, promover la innovación y preocupación por el bienestar de las personas.

En contraste, el actor "A8" no representa un líder potencial en ninguna dimensión de amistad y liderazgo; y el actor "A9" tampoco es percibido como "sólo interviene cuando los problemas se vuelven serios". Estos dos actores son los únicos que muestran algún nivel de "Laissez-Faire", considerado como no-liderazgo.

\section{Discusión y Conclusiones}

Los aspectos de liderazgo que predominan son el clima de "respeto y confianza", el "entusiasmo de los participantes" y la "preocupación por el bienestar de las personas"; inclusive en niveles mayores a los vínculos de "amistad" y la visión que los miembros del grupo son un "ejemplo a seguir". Es muy probable que la naturaleza de la organización, con fines sociales y con trabajo voluntario, haya sido determinante en captar jóvenes voluntarios con un perfil que transmite respeto, confianza, entusiasmo y solidaridad.

El análisis también permitió identificar con claridad el rol de cada líder en las distintas dimensiones de amistad y liderazgo. Se identificaron tres líderes que son los más amigos y se preocupan por el bienestar de los demás. Pero de los tres, uno de ellos destaca por transmitir "mayor respeto y confianza" y es "un ejemplo a seguir"; mientras que los otros dos destacados líderes son los más "entusiastas y optimistas" y "promueven la innovación y creatividad". Un cuarto actor asoma como líder en "amistad", "entusiasmo", "promover la innovación" y "preocupación por el bienestar de las personas", aunque en menor grado que los tres principales líderes. Por el contrario, se identificaron dos actores que no presentan características de líder, muestran algún nivel de no-liderazgo "Laissez-Faire".

Como se evidencia en el presente estudio, el análisis de redes sociales permite identificar el rol de cada uno de los actores en cada dimensión. Permite identificar cuáles son los principales líderes, cuáles son los estilos de liderazgo predominantes, qué dimensiones de liderazgo requieren mayor desarrollo, qué personas de la red y en qué aspectos específicos de liderazgo se presentan aspectos negativos, qué actitudes y comportamientos deben fortalecerse y promoverse, entre otros aspectos que constituyen información relevante para la toma decisiones en la gestión de las organizaciones.

La presente investigación permite mostrar las bondades del análisis de redes aplicado al tema de liderazgo. Se evidencia que el análisis de redes no sólo puede aplicarse a interacciones sociales como las relaciones de amistad u otras, sino también es aplicable para analizar los estilos de liderazgo. El análisis de redes constituye una herramienta alternativa o en todo caso complementaria a otros cuestionarios comunes para medir los estilos de liderazgo. El aporte de análisis de redes es que permite no solamente identificar los estilos de liderazgo predominantes sino también la estructura 
de la reden su conjunto y el rol específico de cada individuo en cada uno de las dimensiones de liderazgo analizadas.

\section{Referencias bibliográficas}

Almería, U. d. (2010). (Molero, Fernando; Recio, Patricia; Cuadrado, Isabel) Recuperado el 8 de abril de 2014, de Liderazgo transformacional y liderazgo transaccional: un análisis de la estrcutura factoria del MLQ en una muestra española.

Bass, B., \& Avolio, B. (1995). Multifactor Leadership Questionnaire (3era. ed. ed.). Palo Alto: Mind Garden.

Bass, B (1985), "Leadership and Performance Beyond Expectations", New York: Free Press; Bass, B. (1990), "From transactional to transformational Leadership: Learning to share a vision", en R.Steers, y G.

Bass, B.M.; Riggio, R.E. (2006), “Transformational leadership”, Mahwah, NJ: Lawrence Erlbaum Associates.

Bigley (Eds.), Motivation and Leadership at Work, pp. 346-354, New York: McGrawHill; Burns, J.M. (1978), "Leadership”, New York, NY: Harper \& Row.

Borgatti, S., Everett, M., \& Freeman, L. (2002). Unicet 6 for Windows: Software for Social Network Analytic.

Bracho, O., García, J., \& Jiménez, E. (marzo de 2012). Universidad del Zulio. Recuperado el 7 de abril de 2014, de Factores de liderazgo transformacional en contralorías municipales del estado Zulia: http://www.publicaciones.urbe.edu/index.php/coeptum/article/viewArticle/1589/3029

Kausel, E., \& Manzi, J. (2010). Análisis de las propiedades métricas de un cuestionario basado en la teoría tridimensional de liderazgo. Estudios de Administración , 17 (1), 51-87.

Lugo Morin, D. R. (2011). Análisis de redes sociales en el mundo real: guía inicial. Revista de Estudios Sociales , 38, 129-142.

Lupano Perugini, M. L., \& Castro Solari, A. (2008). Universidad del Palermo. Recuperado el 5 de abril de 2014, de Estudios sobre el liderazgo. Teorías y evaluaciones.

Mendoza Torres, M. R. (2008). Aproximación al liderazgo desde la caracterización de redes sociales presentes en el aula. Revista de la Facultad de Ciencias Económicas de la Universidad Militar Nueva Granada , XVI (2), 141-160.

Monsalve Moreno, M. (11 de diciembre de 2008). Análisis de redes sociales: un tutorial. Recuperado el 15 de enero de 2014, de http://homepage.cs.uiowa.edu/ mmonsalv/bio/SNA.pdf 
Munaf, S. (2011). Relationship of Transformational, Transactional and Passive/Avoidant Management Style with Achievement Motivation of Teaching Faculties of Selected (Vol. 3). Karachi, Pakistan: 2010 International Conference on Ebusiness, Management and Economics.

Nader, M., \& Castro Solano, A. (2007). Diferencias en los estilos de liderazgo entre líderes de alto y bajo nivel. Un estudio comparativo. Perspectivas en Psicología , 4 (1), 51-58.

Sanz Menéndez, L. (Julio de 2003). Análisis de redes sociales: o cómo representar las estructuras sociales subyacentes. Recuperado el 15 de marzo de 2013, de Unidad de Políticas Comparadas (CSIC). Documento de Trabajo 03-07: http://digital.csic.es/bitstream/10261/1569/1/dt-0307.pdf

Sternberg, J., Antonakis, \& Ciancio. (2004). The Nature of leadership . CA: Thousand Oaks.

Universidad Militar Nueva Granada. (2006). El Liderazgo Transformacional, Dimeniones e Impacto en la Cultura Organizacional y Eficacia de las Empresas (Vol. X IV). (e. C. Red de Revistas Científicas de Amercia Latina, Ed.) Nueva Granda, Colombia. 